\section{Was schützt vor Kuhmilchproteinallergie?}

W ie einer Kuhmilchproteinallergie bei Neu- und Frühgeborenen vorgebeugt werden kann, erläuterte die Allergologin Dr. Anne Des Roches aus Montreal, Kanada, bei einem Satellitensymposium im Rahmen der European Academy of Paediatric Societies in Kopenhagen. Solch eine Allergie entwickeln $2 \%$ der pädiatrischen Population, 15\% der betroffenen Kinder verlieren sie nie.

Untersuchungen zufolge ist das Risiko einer Kuhmilchproteinallergie in dieser Altersgruppe weniger mit einer vor- zeitigen Geburt oder niedrigem Geburtsgewicht assoziiert als mit Asthma oder Nahrungsmittelallergien der Mutter. Des Roches zeigte eine Vorsorge- und Therapieoption auf: „Diskutiert wird eine Präventionsdiät während Schwangerschaft und Stillzeit." Da es an zuverlässigen Studien zu dieser Diät mangele, bestehe Uneinigkeit über deren Wert. Das Meiden von Kuhmilch während des ersten Lebensjahrs sei aber mit einem signifikanten Rückgang der Kuhmilchproteinallergie assoziiert. „Fand diese Präventionsmaß- nahme in der Vergangenheit noch wenig Beachtung, ist ihr Einfluss heute nicht mehr zu vernachlässigen “, fügte Des Roches hinzu. Sie befürwortete die Gabe von hypoallergenem Muttermilchsupplement mit extensiv hydrolysiertem Protein bei Frühgeborenen und von hypoallergener Formula wie Nestlé Beba ${ }^{\circledR} \mathrm{HA}$ bei Neugeborenen in den Fällen, wo die Kinder aus atopischen Familien stammen und somit ein höheres Risiko tragen, eine Kuhmilchproteinallergie zu entwickeln.

red

Nach Informationen von Nestlé Nutrition, Frankfurt/Main

\title{
Allergopharma mit neuem Internetauftritt
}

D as Unternehmen Allergopharma aus Reinbek bei Hamburg präsentiert sich bereits seit Dezember 2010 unter www.allergopharma.de mit einer optisch wie inhaltlich überarbeiteten Internetpräsenz. Ein erster Blick auf die neue Startseite liefert Ärzten und Apothekern sofort aktuelle News aus der Allergologie. Allergiekranke können sich mit der Pollenfluganzeige unmittelbar über die aktuelle Belastung in ihrer Umgebung informieren. Die übersichtliche Menüauswahl des neuen OnlineAngebots erlaubt dann eine schnelle

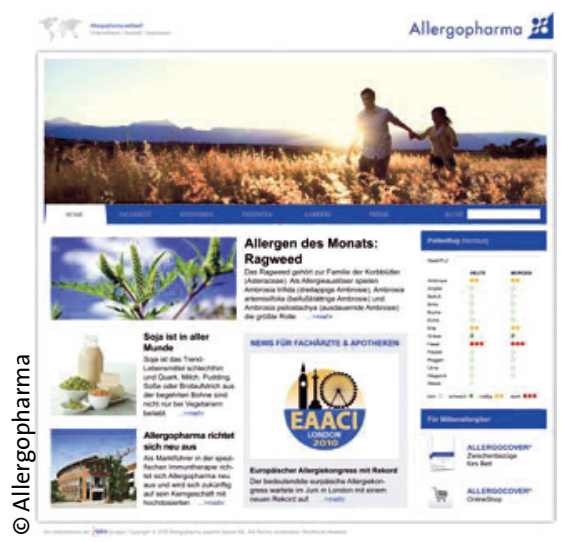

Die neu gestaltete Internetpräsenz www.allergopharma.de lädt Ärzte, Apotheker und Patienten gleichermaßen dazu ein, sich rund um das Thema Allergie zu informieren.
Orientierung über die weiteren Inhalte und Angebote.

Ärzte finden auf der Website ausführliche Informationen $\mathrm{zu}$ grundlegenden Aspekten der IgE-vermittelten Allergie, zu deren Diagnostik und zur spezifischen Immuntherapie. Zusätzlich können sie auf verschiedene Serviceleistungen zugreifen: Praxisbroschüren, Formulare und Produktinformationen sind per Mausklick abrufbar. Online angefordert werden kann auch der neue Therapie-Organizer THEO, ein Tool zur Förderung der Compliance bei der spezifischen Immuntherapie. Für weiterführende Fragen enthält die Website einen Überblick über das Beratungsangebot von Allergopharma mit den Durchwahlen der Ansprechpartner für die medizinische Beratung sowie für die Bestellung von Therapeutika und Diagnostika.

Für Patienten bietet die neue Internetpräsenz von Allergopharma Wissenswertes zum richtigen Verhalten bei einer Allergie sowie zu Therapiemöglichkeiten. Bei Fragen zu Allergieauslösern erweist sich das "Lexikon der Allergene“ als hilfreiche Unterstützung für erste Erläuterungen. Außerdem steht auch für Betroffene eine große Auswahl an Broschüren zum Download bereit.

Nach Informationen von Allergopharma, Reinbek
Konzentration auf das Wesentliche

Mit dem Ziel, sich zukünftig ganz auf das Geschäftsfeld spezifische Immuntherapie konzentrieren zu können, hat die Allergopharma Joachim Ganzer KG Ende des letzten Jahres einen Vertrag über den Verkauf ihrer Geschäftseinheit In-vitro-Allergiediagnostik an die Firma Omega Diagnostics PLC unterzeichnet. Dadurch wird das in Deutschland sehr erfolgreiche IgETestsystem von Allergopharma an die neu gegründete Firma Omega $\mathrm{GmbH}$ mit Sitz in Reinbek bei Hamburg übertragen. Omega übernimmt die gesamte Produktpalette, tritt in alle bestehenden Verträge ein und beschäftigt alle Mitarbeiter weiter. Eine Internationalisierung und Weiterentwicklung der derzeitigen Allergietest-Plattform sind geplant. Omega wird durch eine enge Zusammenarbeit die Kompetenzen von Allergopharma im Bereich Allergie nutzen. So wird das Unternehmen seine benötigten Allergene von Allergopharma beziehen. Für die Allergologen ist damit die gleiche Qualität wie in der Vergangenheit sichergestellt.

Joachim Ganzer, Geschäftsführender Gesellschafter von Allergopharma: „Ganz wichtig war uns, dass die Arbeitsplätze in Reinbek und in der Geschäftseinheit gesichert sind. Wir freuen uns sehr, dass dies gelungen ist. Mit dem Verkauf können wir jetzt die notwendigen Ressourcen für die Weiterentwicklung unserer rekombinanten Allergen-Pipeline noch verstärken." Allergopharma plant, die ersten rekombinanten Allergenprodukte 2014 in Europa einzuführen.

Nach Informationen von Allergopharma, Reinbek 